\title{
Against the Excesses of Quantum Gravity: A Plea for Modesty
}

\author{
Erik Curiel ${ }^{\ddagger}$
}

I argue that all current research programs in quantum gravity conform to the 17 th century hypothetico-deductive model of scientific inquiry, perhaps of necessity given the current state of technology. In so far as they do not recognize and advertise the shortcomings of the research method they use, they do a disservice to the integrity of science, for the method admits of far less certainty accruing to its products than one would be led to believe by the pronouncements of researchers in the area.

1. The Mechanical Philosophers of Quantum Gravity. In 1672, Isaac Newton published in the Transactions of the Royal Society his theory of the structure of light rays (Newton [1671/1672] 1958). It was not understood by even his most brilliant contemporaries, scientific luminaries such as Robert Hooke and Christian Huygens (see Hooke [1672] 1757/1958 and Huygens [1673a] 1958, [1673b] 1958; for Newton's responses to their respective criticisms, see Newton [1672a] 1958, [1673a] 1958, [1673b] 1958). The primary obstacle hindering their understanding was their conception of proper scientific methodology, the hypothetico-deductive method as applied solely in the theater of the mechanical philosophy. Neither Newton's account of the derivation of his theory nor the theory itself conformed to this conception of science, much to the discredit of that conception. ${ }^{1}$

† Send requests for reprints to the author, 401 Steiner St, \#2, San Francisco, CA 94117; email: erik@ilium.org.

$\ddagger$ I thank Rob Clifton, Matt Frank, John Hunter, Carlo Rovelli, Eric Schliesser, and Michael Strevens for penetrating comments on an earlier draft of this paper. It would have been a far poorer work than it is without their input; I remain responsible for its errors and defects. I am particularly grateful to Carlo for responding to my mildly hysterical prose with good humor, grace, patience, and (best of all) invaluable insight on virtually every aspect of the issues discussed.

1. See Stein n.d.a, n.d.b for a thorough account of the incident, its historical context, and the particular aspect of it I am referring to.

Philosophy of Science, 68 (Proceedings) pp. S424-S441. 0031-8248/2001/68supp-0034\$0.00 Copyright 2001 by the Philosophy of Science Association. All rights reserved. 
In the 1990's, quantum gravity came into its own as an accepted, even a sexy field of research in theoretical physics. Except for adherence to the mechanical philosophy, all the major research programs in quantum gravity today do conform to the conception of science championed by Newton's contemporaries, much to the discredit of quantum gravity.

In this paper I shall make the case for this claim, and discuss a few of its unfortunate corollaries. I shall also examine the scientific standing of various programs in quantum gravity as reported by proponents of those programs in order to criticize what I see as their immodesty-unwarranted in light of my arguments in the first part of the paper-which at times seems to cross the border into disingenuity. It is no mere Pauline gripe I have against this immodesty; I think it does real damage to science on many levels. I shall conclude with a few reflections on this matter and on the role, if any, that philosophers of science ought to play in the maintenance of working science's integrity.

2. The Hypothetico-Deductive Method Illustrated and Criticized. In the preface to his Treatise on Light, Christian Huygens spells out as clearly as one could wish the essentials of the hypothetico-deductive method of science:

There will be seen in [this work] demonstrations of those kinds which do not produce as great a certitude as those of Geometry, and which even differ much therefrom, since whereas the Geometers prove their Propositions by fixed and incontestable principles, here the Principles are verified by the conclusions to be drawn from them; the nature of these things not allowing of this being done otherwise. It is always possible to attain thereby to a degree of probability which very often is scarcely less than complete proof. To wit, when things which have been demonstrated by the Principles that have been assumed correspond perfectly to the phenomena which experiment has brought under observation; especially when there are a great number of them, and further, principally, when one can imagine and foresee new phenomena which ought to follow from the hypotheses which one employs, and when one finds that therein the fact corresponds to our prevision. (Huygens [1690] 1912/1962, vi-vii)

In order to investigate some physical entity scientifically, one first formulates a hypothesis about it, consisting of a set of propositions serving as a formal model of the entity. One then deduces consequences from the hypothesis, describing the behavior of the entity under certain experimentally replicable conditions, performs the appropriate experiments, and checks to see whether the outcome of the experiment matches the predictions of one's hypothesis: the more times that one performs this trick 
successfully, the higher the probability of correctness that accrues to one's hypothesis.

Where, though, do the initial hypotheses come from? What constraints, if any, can one place on them? What ideas can one legitimately use to inspire one in constructing them? Past experimentation and hypothesizing on related topics are two obvious sources of constraints and ideas, and Huygens does freely avail himself of them, rightfully so. There is, however, for Huygens, an even more important source:

[I]n the true Philosophy, ... one conceives the causes of all natural effects in terms of mechanical motions. This, in my opinion, we must necessarily do, or else renounce all hopes of ever comprehending anything in Physics. (Huygens [1690] 1912/1962, 3)

Because, to Huygens, the only conceivable form and action of physical substances were those portrayed in (some variant of) the mechanical philosophy, all hypotheses forwarded in an attempt to explain any physical phenomena whatsoever had to conform to it.

The hypothetico-deductive method does not require one to have a fixed set of principles, metaphysical or otherwise, by which one is to judge the legitimacy of possible hypotheses before using them in deductions; in practice, however, the two naturally, almost unavoidably go together. Before commenting on this view of the proper working of science, I turn to Newton.

The incident I wish briefly to rehearse is the dispute Newton had with several of his most distinguished contemporaries upon the publication in 1672 of his doctrine of the structure of light. Newton demonstrated unequivocally: that sunlight consists of an innumerable number of simple light rays differing from each other in their refrangibility relative to a given medium of propagation; that the refrangibility associated with a particular simple ray for a given medium of propagation is a primitive property of that ray in the sense that no manipulation of the ray Newton could contrive would change it (it was this fixity of the refrangibility that led Newton to think of these rays as the "simple" components of the more "complicated" ray of sunlight); ${ }^{2}$ that when a simple ray exhibits a particular refrangibility, it also always exhibits a particular correlated colorific character, viz. the appearance to a normal observer under normal conditions of a particular color when the simple ray falls on a white surface, always the same color for a given refrangibility; and that light from all sources he could find to experiment on has the property of being composed of simple rays of fixed refrangibility, if it is not itself in the first place such a simple ray. In modern terminology, Newton discovered that an adequate 
mathematical representation of any light ray, relative to the experimental technique available to him, has (crudely speaking) a unique Fourier decomposition; that the refrangibility of individual components of the Fourier composition - a ray of light of a single pure frequency - depends on the frequency of the light; that (visible) light of a single frequency tends to produce only one color, always the same, to a normal observer under normal conditions; and that the frequency of a light ray cannot be changed (at least, again, not by any experimental methods known at the time to Newton).

As astonishing as these results were in themselves, and as difficult as they were for many of his contemporaries to digest and accept, in the end not the results themselves but rather Newton's method of arriving at and of formulating them caused the most confusion.

In his reply to a letter from the French Jesuit Ignatius Pardies, in which Pardies criticized his doctrine of light, Newton makes his clearest statement describing the essence of this method, which suggests some clues as to why Newton's contemporaries may have greeted his doctrine with uncharacteristic confusion; in the process, he also offers a trenchant criticism of the hypothetico-deductive method of scientific investigation:

[T] he doctrine which I explained concerning refraction and colours, consists only in certain properties of light, without regarding any hypotheses, by which these properties might be explained. For the best and safest method of philosophizing seems to be, first to inquire diligently into the properties of things, and establishing those properties by experiments and then to proceed more slowly to hypotheses for the explanation of them. For hypotheses should be subservient only in explaining the properties of things, but not assumed in determining them; unless so far as they may furnish experiments. For if the possibility of hypotheses is to be the test of truth and reality of things, I see not how certainty can be obtained in any science; since numerous hypotheses may be devised, which shall seem to overcome new difficulties. (Newton [1672] 1958, 106)

Newton's deduction of his conclusions and his statement of them do not depend on any prior hypotheses in the sense that they do not depend on any particular hypotheses one may have about the physical structure of light rays-whether one postulates that they are waves in some ætherial medium or that they are particles or whether one postulates nothing about their physical microstructure at all, Newton's propositions not only make sense but hold true, for they did nothing more than describe experimentally verifiable properties of light. Newton had, moreover, no thought during that derivation of trying to explain the observed behavior by this or that hypothesis; that maneuver, if performed at all, would come only after the 
fundamentals of the behavior of light under various experimental circumstances were captured by a series of propositions independent of any particular hypothesis about its microstructure. To a devotee of the method of science articulated by Huygens, such a feat was incomprehensible.

The superiority of Newton's method, when available, is clear. The making of later verified predictions on the basis of a particular hypothesis gives precious little confirmatory support to any grand hypothetical structure in toto; at most it can give slight support, if even that, to those particular bits of the hypothetical structure that played a role in producing the particular prediction. One who is clever enough will always be able to construct competing hypotheses that yield the same predictions, and those hypotheses will then acquire as much or as little confirmation as the original, with the implication that such tests imbue no particular hypothesis with any definite advantage, as Newton's penetrating remarks suggest. Newton's parting shot at the hypothetico-deductive method, though, hits hardest: when experimental results do not bear out any given hypothesis, the temptation will be strong simply to fiddle with the hypothesis until it gives the right results. The danger that the researcher will blind himself to promising new routes of investigation in the process - will willfully ignore the world's attempts to convey to him a fundamental inadequacy in the representational scope of his dearly held hypothesis-is all too clear. Indeed, when deployed in the absence of any constraining experimental data, it is difficult to see how the hypothetico-deductive method can yield genuine physical knowledge or understanding of the world at all, rather than merely some small insight into the psychology of the researcher championing the hypothesis.

3. When Contact with the World Is Wanting. Much has been made of the fact that it will be difficult to test theories of quantum gravity experimentally, in light of the extremely large scales of energy and extremely small scales of spatiotemporal intervals at which it is expected that characteristic features of these theories will reveal themselves, scales far beyond any that humans will be able to probe in the foreseeable future. This fact has been used to argue that we ought to be wary about the scientific stature accorded these theories. I agree with this point in a limited way; it is not for me, however, the primary cause of concern about theories of quantum gravity.

In a remarkable recent paper, Carlo Rovelli, an eminent researcher in the field of quantum gravity, makes the following observation:

Of course, we have little, if any, direct empirical access to the regimes in which we expect genuine quantum gravitational phenomena to appear. Anything could happen at those fantastically small distance 
scales, far removed from our experience. Nevertheless, we do have information about quantum gravity, and we do have indications on how to search for it. In fact, we are precisely in one of the very typical situations in which good fundamental theoretical physics has been working at its best in the past: we have learned two new extremely general "facts" about our world, QM and GR, and we have "just" to figure out what they imply, when taken together. The most striking advances in theoretical physics happened in situations analogous to this one. (Rovelli 1999, 2)

Although I heartily endorse much of the spirit of this paper and many of its particular claims, I cannot agree with this analysis of the present situation in theoretical physics and its relation to possible historical precedents.

All great revolutions in physics so far have taken place under the impetus of masses of seemingly inter-related experimental evidence that could not otherwise have been explained by the best going theories of the day. ${ }^{3}$ Even Newton's doctrine on light fits this pattern: the then received version of Snell's law could not account for the fact that a circular pencil of sunlight falling obliquely on a triangular glass prism should be dispersed into an elongated spectrum. The revolutions themselves all have the following character as well: they consisted of a set of propositions that encapsulated all the known, seemingly inconsistent experimental data in a set of propositions describing the properties of the experimental phenomena in a way that did not depend at all on particular hypotheses about possible microstructures underlying the phenomena, all in the spirit of Newton as well. They thus avoided the tempting snare set by use of the hypotheticodeductive method: reliance on one's own idiosyncratic philosophical predilections - which the physical world has no obligation to satisfy-as the court of final appeal in judging the adequacy of scientific propositions.

Galileo had his experimental observations, encapsulated in the proposition that all free bodies fall with the same acceleration, a fact that could not be accounted for by then prevailing Aristotelian notions; these led him to postulate his law of falling bodies.

Newton had Galileo's law of falling bodies, Huygens' researches on pendulums and circular motion in general, and Kepler's planetary laws, themselves up to that point merely phenomenological laws inferred from Tycho Brahe's and Kepler's own astronomical observations; on the basis of these, as well as on that of myriad other empirical considerations (e.g., the acceleration of the Moon about the Earth), Newton deduced his law

3. Copernicus's bold hypothesis of heliocentrism may be a counterexample to this claim.

This is a tricky historical incident to make scientific sense of. 
of universal gravitation, taking pains all the while to emphasize that his theory only described the formal laws that bodies' motions conform to, and did not feign hypotheses about the causes of their always following motions determined by the law of gravitation.

Maxwell had the wealth of Faraday's experimental researches on electrical and magnetical and optical phenomena, as well as a vast body of experimental data and phenomenological laws due to a host of other able researchers, to suggest directly a deep connection between electricity, magnetism, and light; Maxwell himself emphasized that the final breakthrough came only after he had relinquished attempts to provide exact microstructural models of the postulated electromagnetic æther and instead tried only to formulate a system of mathematical propositions that would adequately encode all known behavioral characteristics of electromagnetic phenomena (see Maxwell [1864] 1965, passim).

Einstein had, for his postulation of special relativity, Fizeau's experiments describing the effect that a moving medium has on the propagation of light in that medium; Hertz's remarkable experiments demonstrating the existence of electromagnetic radiation in otherwise empty space as a spectacular confirmation of Maxwell's theory; the Michelson-Morley experiment, which signally failed to detect any motion of the Earth through an ambient æther; centuries' worth of observations supporting the Galilean insight that there is no detectable physical difference among different inertial frames of reference; and the invariance of electromagnetic phenomena with respect to the Lorentz transformations rather than the expected Galilean transformations.

Planck, Einstein, Bohr, Heisenberg, et al., had the failure of classical theory to describe the correct behavior of blackbody radiation; the related failure of classical theory to explain the experimentally determined behavior of the specific heat of a material body as decreasing monotonically with decreasing temperature; its failure to account for the photoelectric effect; the unexpected discreteness of atomic and molecular spectral lines; the Compton effect; the Franck-Hertz experiments demonstrating the quantization of the kinetic energy of free electrons; and so on.

Finally, for general relativity, Einstein had, in addition to both the well documented, anomalous precession of the perihelion of Mercury's orbit and the centuries' worth of observations confirming the equivalence principle, the manifest incompatibility of the instantaneous action at a distance postulated by Newton's theory of gravitation with the prohibition of such phenomena by special relativity. ${ }^{4}$

What comparable body of seemingly related but otherwise conjointly

4. This emphasis on experimental data represents an unorthodox interpretation of the provenance of general relativity, which I plan to defend in detail in a future paper. 
inexplicable experimental data drives the attempts to unify quantum mechanics and general relativity into a single quantum theory of gravity? None. All experimental evidence describing each known department of quantified physical phenomena is in harmony with whichever of the two theories is most appropriately used to model that department. Contrary to the folklore, moreover, there is no manifest contradiction between the two theories themselves.

For a good example of the insouciance with which claims of the inconsistency of the two theories are treated - a sure sign of mythology - one need look no farther than the first sentences of a very recent survey article by Juan Maldacena, one of the bright stars in string theory's firmament:

Our present world picture is based on two theories: the standard model of particle physics [i.e., quantum field theory] and general relativity, the theory of gravity. These two theories have scored astonishing successes. It is therefore quite striking when one learns that this picture of the laws of physics is inconsistent. The inconsistency comes from taking a part of theory, the standard model, as a quantum theory while the other, gravity, as a classical theory. (Maldacena 2000, 1)

I confess I do not understand this claim at all. Until some content is given to a theory's being "quantum" or being "classical," the inconsistency cited is strictly adjectival. I strongly suspect, moreover, that no trivial definitions of "quantum" and of "classical" could yield the imputed inconsistency: inconsistencies among physical theories arise either when they make conjointly inconsistent predictions or when they presuppose conjointly inconsistent principles. Relativistic quantum field theory, however, in its present, most perfected form, the Standard Model, places no demands on the character of spacetime in general or on that of gravitational phenomena in particular that general relativity cannot cope with; a fortiori none of its principles or predictions conflict with any proposition of general relativity. The problems that plague attempts to formulate nonlinear quantum field theory on "classical" curved backgrounds, for example, are all purely technical in nature, not based on any seemingly irreconcilable rift between the two theories or between one of the theories and any experimental data (see, e.g., Wald 1994, passim). General relativity does not pretend to care about the specific character, quantum or classical, of the physical laws formulated on its spacetimes, as the mathematically rigorous formulation of linear quantum field theory on curved backgrounds attests to. The superposition principle, for instance, arguably the most "quantum" of all quantum mechanical principles, controverts no fundamental tenet of general relativity.

So far from there being any experimental catastrophe crying out for theoretical innovation, we are in the truly remarkable position of having 
two fundamental theories confirmed to accuracies beyond imagining by all experimentation conducted to date, as has not been the case in any of the previous periods of great revolution in physics. There exists no physical phenomenon well characterized by experiment that cannot be accurately described by one of the two theories, and no physical phenomenon that suggests that one of the two is correct to the detriment of the other's accuracy. ${ }^{5}$

The mettle of physics by itself surely does demand the attempt to integrate these two spectacularly successful theories into one unified theory; I am the last to dispute this. My point is that nothing more than this desire to fortify physics' mettle currently drives the attempts. There are no propositions describing the behavior of any seemingly quantum gravitational phenomena drawn directly from experimentation to guide intuition and to constrain technical extrapolation. The experimental chasm gapes so deep and wide between, on the one side, the features of quantum mechanics and general relativity firmly founded on experiment and, on the other, the sort of physical situations in which there is reason to suspect that peculiarly quantum gravitational phenomena will appear-e.g., the near vicinity of a black hole whose radius is on the order of the Planck scalethis chasm gapes so wide as to preclude almost no suggestion of a research program and no particular formulation of one from falling in. Questions breed with Malthusian ferocity, and we are at vertiginous liberty to answer them as we will, or even to reject them, for no reasons based on well grounded physical knowledge. What aspects of spacetime should we attempt to include in a quantum theory? Metric structure? Affine structure? Conformal structure? Projective structure? Differentiable structure? Topological structure? Point-set structure? How ought one give a quantum theory of any of these? What may it mean to give a quantum theory of any of these? And so on. We are wholly within a realm where nothing more and nothing less guides hypothesizing than philosophical predilections of the same sort, and of the same standing, as Huygens' for the mechanical philosophy; and where the only way forward is to propose such hypotheses in the absence of any relevant, constraining experimental data, deduce consequences from the hypotheses, and hope one day to have the technological wherewithal to compare these deductions to experiments-which constitutes the hypothetico-deductive method of practicing science.

5. Certain astrophysical phenomena, such as the mysterious gamma ray bursts observed in recent years, might be thought a counterexample to this claim, but they are not. Since we have no direct knowledge of the structure of the systems producing these phenomena, and therefore no method in which we have any great confidence for applying current theory in an attempt to model the systems and so explain the phenomena, such phenomena can neither support nor controvert current theory. 
4. The Present Standing of Theories of Quantum Gravity. In order to evaluate the scientific status of theories of quantum gravity properly, one must, among other matters, understand what such theories hope to accomplish and how far along the road to these goals they are. To quote Maldacena again:

The challenges we face can be separated, in degree of difficulty, in the following three:

I. Formulate an internally consistent theory of quantum gravity. By this we mean a theory which reduces at low energies, $E<<$ $10^{19} \mathrm{Gev}$, to general relativity but in which we can perform quantum calculations to any order we wish. This theory should solve some fundamental problems with quantum gravity such as explaining the origin of black hole entropy, etc. These are questions about gravity which do not involve directly the fact that we also have the particle physics that we see in nature.

II. Be capable of incorporating the Standard Model. So the theory should be such that at low energies it can contain chiral gauge fields, fermions, etc.

III. Explain the Big-Bang and the parameters of the Standard Model. We should understand the resolution of the initial singularity in cosmology and we should understand why we have the Standard Model. We should understand how the Standard Model parameters arise, which parameters are related, and which parameters (if any) arise as a "historical" accident.

We now have a theory, called string theory (or M-Theory), which has been able already to provide a solution to the first two challenges. . . . We know several limits and aspects of the theory but we still do not know the fundamental axioms of the theory that would enable us to approach the third challenge. (Maldacena 2000, 2) ${ }^{6}$

Note the glaring lack of mention of any contact with motivating experimentation; more troubling for me is the absence of any awareness that this is problematic, not only with regard to the making of predictions, but even more so with regard to a theory's being based (or not) on firmly grounded knowledge derived directly from experiment.

6. I quote at such length in order the better to substantiate my criticisms, which perhaps will seem unduly harsh or rash to one who has not read much of the literature. Precisely the same sentiments about string theory were voiced by Edward Witten, the doyen of string theory, in a talk he gave at a symposium on quantum gravity early in the summer of 1998 at Rutgers University, and later in informal conversation at a weekend retreat held after the symposium. I regret I do not have the transcript of that talk from which to quote. 
How ought one judge his claim that string theory has already met the first two listed challenges? I regret that I do not have the technical competence to judge the success of string theory in incorporating the Standard Model. I am, however, competent to judge its success vis-à-vis the first challenge: it does not meet it. As Maldacena admits explicitly on page 6 of the same article, string theory is at the moment formulated only as a perturbation theory on Minkowski spacetime; he even implies on page 8 that conjectural modifications to string theory up to this point have consisted only of reformulations on fixed, curved background spacetimes"classical" ones! - which to my knowledge is true. The most charitable one can be is to say that Maldacena answers in the negative to most of the questions mooted at the end of the preceding section about possible desiderata of a theory of quantum gravity; though this does make it even more difficult to know what to make of his claim that quantum mechanics and general relativity are inconsistent in so far as the one is a quantum and the other a classical theory.

I shall not have space enough to discuss Maldacena's plaintive remark that further work on the third challenge will be impossible until the "fundamental axioms" of the theory are known; a nod toward its redolence of one part of what was defective about Huygens' method will have to suffice. I instead want to focus for the moment on that part of Maldacena's first challenge that mentions the explanation of black hole entropy, identified here as a "fundamental problem of quantum gravity." The loudest crows of triumph in the quantum gravity arena have arisen over the success of various theories in computing, from 'first principles', the BekensteinHawking entropy attributed to black holes on the basis of semi-classical calculations modeling quantum fields on a classical, curved, relativistic background. Indeed, it was the computation of this result in a special case by Strominger and Vafa in 1996 that was single-handedly responsible for reviving the then flagging reputation of string theory (see Strominger and Vafa 1996).

To give the flavor of the importance accorded these results, based on the respect accorded the Bekenstein-Hawking formula in the theoretical physics community, I offer representative quotations from leaders of the two primary research programs in quantum gravity today:

The fact that [black holes] are thermal objects raises very interesting and very important theoretical puzzles, [sic] solving these puzzles is

7. It is not clear to me why Maldacena claims that this is an issue that does not depend on any knowledge we have of particle physics. The association of a multiple of a black hole's surface area with entropy was clinched by Hawking's semi-classical calculation, based on quantum field theory, that black holes emit blackbody thermal radiation. See Hawking 1975. 
one of the challenges of a theory of quantum gravity. (Maldacena 2000, 6; italics are mine)

[T] he detailed information about the eigenvalues of these [area] operators [in loop quantum gravity] has interesting physical consequences, e.g., to the process of Hawking evaporation of black holes. (Ashtekar 1999, 3; italics are mine)

What is striking is that these two physicists-and not only these, but almost every one I have read in the literature-treat the thermal radiance of black holes as a physical fact, on par, say, with the hyperfine structure of the spectrum of the Hydrogen atom, a fact, that is to say, which is a thing not only of theoretical calculation but of experimental observation. Its true status, however, was inadvertently advertised in a remark made by Leonard Susskind in a recent talk he gave on string theory, in which he used the fact that string theory allowed one to calculate the BekensteinHawking entropy formula by a counting of black hole microstates to argue for the high scientific merit of string theory: "No one disputes [the fact of thermal radiance by black holes and the Bekenstein-Hawking entropy formula]; this is not a matter of contention among theorists" (Susskind 1999; italics are mine, in so far as one can say this of spoken words). The derivation of the Bekenstein-Hawking entropy formula by the counting of microstates in this or that theory of quantum gravity, impressive and intriguing as it may be in many ways, cannot serve as a demonstration of the scientific merit of a theory of quantum gravity, for the BekensteinHawking entropy formula itself has no empirical standing. There is not the slightest shred of empirical evidence that directly supports or controverts it. ${ }^{8}$

Neither was the particle theory of light a matter of contention among theorists after the time of Newton until the beginning of the $19^{\text {th }}$ century, although no experimental evidence buttressed the particle theory so completely as to exclude utterly Huygens' formulation of the wave theory. There was no direct experimental evidence that warranted the firm conclusion that light was particulate in a mechanical sense-as should be

8. To his credit, among the many researchers on quantum gravity whose publications I have read in which this issue is discussed, Rovelli is the only one to admit that the Bekenstein-Hawking entropy formula does not have any empirical support:

[Q]uantum field theory in curved spacetime is by now a reasonably established theory ... predicting physical phenomena of remarkable interest such as particle creation, vacuum polarization effects, and Hawking's black hole radiance. . . . To be sure, there is no direct nor indirect experimental observation of any of these phenomena, but the theory is quite credible as an approximate theory, and many theorists in different fields would probably agree that these predicted phenomena are likely to be real. (Rovelli 1998, 10) 
expected, since it is not! The consensus of theorists in the absence of direct experimental evidence does not suffice for investing a hypothesis with any scientific credibility.

If in some sense the semi-classical approximation is a 'natural' extension of well established quantum mechanical technique into the arena of curved spacetime backgrounds, moreover, and so gains some warrant from the faith we have in this set of techniques for accurately representing certain aspects of the structure of the physical world, then even so the fact that various theories of quantum gravity reproduce the BekensteinHawking entropy formula cannot be taken as confirmatory of those theories in the slightest. Reproducing well established results is the absolute minimum required of any theory that may have a claim to physical adequacy. To argue otherwise is again to rely on the $17^{\text {th }}$ century hypotheticodeductive model of scientific method, which, I have argued, has not been used in any previous major scientific revolution and is not appropriate for use in any future ones.

I for one am significantly more impressed by the calculation of the spectrum of area and volume operators in loop quantum gravity than I am by the derivation of the Bekenstein-Hawking entropy formula in any theory - these spectra represent physical quantities that one can envision being probed in the not-so-distant future (see Rovelli and Smolin 1995). The fact that this is only a prediction, however, constructed from a very specific hypothetical model of the microstructure of quantum geometry implies that, for reasons mentioned in $\$ 2$ above, even were the spectra measured and found to accord well with the predictions of that theory, only weak credibility at best would accrue to the entire theory, and only marginally stronger credibility would accrue to that part of the theory essential for making the calculation. That the advocates of loop quantum gravity do not explicitly recognize this point I believe stems from a perhaps unintentional adherence to Huygens' scientific methodology.

The attitude taken in numerous publications by Ashtekar, one of the driving forces behind loop quantum gravity, provides evidence for this hypothesis. I find the following passage a particularly poignant example:

[From observation of gamma ray bursts, on the assumption that they are cosmological in origin,] astronomers have already put some very interesting limits on the 'graininess' of geometry. Now the challenge for the theorists is to construct realistic [non-perturbative loop quantum gravity] states corresponding to the geometry we observe on cosmological scales, study in detail propagation of photons on them and come up with specific predictions for astronomers. (Ashtekar 1999, 15) ${ }^{9}$

9. It should also be noted that the claim that the gamma ray bursts are cosmological in origin is itself highly speculative, based more on the fact that theoreticians have so 
Here is one of the first indications I have ever seen that astronomical observations technologically feasible today might be able to provide information about the structure of spacetime at length scales small enough so that one would have sound reason to believe that quantum gravitational effects could well appear, and the theoretician's response is not: let us try to construct a theory of quantum geometry based on the experimental evidence we shall acquire; nor even: let us try to ascertain whether the non-perturbative states of loop quantum gravity are appropriate representations of the possible quantum spacetime structure astronomers are able to glean hints of from their observations. Rather only: let us tweak the pre-existing hypothesized-mechanical!-model to fit the new evidence, and then make predictions.

In a recent review of all major quantum gravity research programs, Rovelli writes, "string theory is today, without a doubt, the leading and most promising candidate for a quantum theory of gravity. It is the theory most studied, most developed and closer [sic] to a consistent and comprehensive framework" (Rovelli 1998, 5). I take issue with this statement: none of the last three attributes Rovelli mentions suffices for rendering string theory "the most promising" of quantum gravity research programs, though "leading" may be granted, so long as this is taken in only a sociological sense. Indeed, my contention is that no contender deserves praise above any other yet (though some may well deserve more blame), because none has, as of yet, any peculiarly scientific merit on which to be judged. Elegance, beauty, consistency, et al., are all arguably necessary merits of a scientific theory, but none of them is peculiarly scientific, and so they cannot on their own, even taken all in concert, be used as criteria for judging the relative scientific merits of theories. The peculiarly scientific merit of a scientific theory is its contact with empirical research, which can take many forms: already existing empirical data may confirm the predictions of the theory; already existing empirical data may suggest the theory; the theory may suggest new avenues of empirical research, the results of which may in turn either support or controvert the theory; and so on. In this sense, no theory of quantum gravity has the slightest bit more of scientific merit than any other, no matter the elegance of the theory, no matter the number or eminence of the physicists working on the theory.

5. A Plea for Modesty. Most of the researchers at work in quantum gravity today are conscientious and scrupulous scientists. The immodest and grandiose claims made by a few, however, about the achievements, indeed the inevitability of correctness of their own research programs can sometimes

far not yet been able to construct any plausible models for a local source than on direct experimental evidence. 
smack of the desperate and delusional. It is instructive to compare the panegyrics delivered on the present state of theoretical physics with the judgment of a particular man on his own work, in which he had more secure reason for pride than any quantum gravity theorist alive today does in his or her own work; which renders him an even more admirable scientific figure than one may have thought, and an example more worthy of emulation in this regard than any other I know. I refer to Newton's remarks, in the preface to his Principia, on possible new lines of research suggested by his method of deriving the theory of universal gravitation:

I wish we could derive the rest of the phænomena of nature by the same kind of reasoning from mechanical principles; for I am induced by many reasons to suspect that they may all depend upon certain forces by which the particles of bodies, by some causes hitherto unknown, are either mutually impelled towards each other, and cohere in regular figures, or are repelled and recede from each other; which forces being unknown, philosophers have hitherto attempted the search of nature in vain; but $I$ hope the principles here laid down will afford some light either to this or some truer method of philosophy. (Newton [1687] 1726/1995), 4; italics are mine) ${ }^{10}$

Although there is much that deserves to be lingered over in this passage, circumstances do not permit this; for the present purpose it is the last, italicized clause that summarizes what is worthy of admiration. That the "mechanical" principles (not in the sense of Huygens' mechanical philosophy) laid down in his three laws of motion paved the way to the theory of universal gravitation, the most striking scientific success up to that time, being the first example of a truly universal scientific law-as Newton himself was well aware - that they did so, did not suffice for Newton to allow himself the luxury of thinking that he had definitively hit upon the key for understanding all aspects of the physical world.

The present day panegyrists of quantum gravity, though having much less in hand of which they can securely boast than Newton had, nonetheless show none of his scruple in judging the merit of their own work. With this immodesty, they present a danger not only to the integrity of the scientific community from the point of view of its own, but perhaps even more so from the point of view of the educated world at large. To the apprentice physicist, they teach the lesson that what passes as real science

10. Many philosophers could learn a valuable lesson in this regard from Newton as well. I invite the reader to compare, for example, the sentiment Descartes expresses in his letter to the Abbé Picot that serves as the preface to the French edition of his Principles of Philosophy, or that of Wittgenstein in the preface to his Tractatus LogicoPhilosophicus. 
can be successfully done in the absence of hard physical data, so long as the promulgator of the 'science' is eminent enough; in light of the fact, moreover, that a physicist can nowadays become eminent on the strength only of producing fantastical theories, cynicism and a disrespect for the basics of sound scientific method will surely result. For the educated layperson, these grandiose claims strengthen and propagate the dangerous myths of the omniscience of science and of the ease and inevitability of its progress and ultimate success in its quest to understand the physical world. ${ }^{11}$

I do not want it thought that I am arguing that theoretical advances in physics can come only under the duress of direct experimental evidence not otherwise explicable, that if we cannot practice Newton's method of science, then we ought not practice science at all. Lagrange's and Hamilton's development of entirely new mathematical frameworks for classical physics, for example, provide beautiful counterexamples to that extreme position. When the 'advances', however, purport to extend the reach of current physical knowledge in radical ways without any direct experimental pressure to do so, one must have some anxiety about their propriety as physical theories until experimental confirmation is forthcoming. Even then, moreover, one must consider whether the forthcoming experimental evidence supports the proposed theory univocally, as it will be able to do only if that theory is not overly hypothetical in Huygens' sense of the term.

There is likely no other method for research to be carried out today on quantum gravity than that presently being employed; although I think the method deficient when compared to one that relies on empirical data as its engine and not its terminus, I do not want to suggest that research on quantum gravity be stopped merely because it must employ a second-best method. What I would have of the physics community is a more honest appraisal of the standing of its efforts in this area in light of the deficiencies of the method that will of necessity be employed. One of the current theories of quantum gravity may indeed turn out to be supported by as of now undreamt of direct experimental confirmation, may turn out to be

11. It has been suggested to me in private correspondence by a few people, in the attempt to mitigate some of my criticism, that the tenor of current claims about the scientific stature of current programs in quantum gravity reflects the note these physicists feel they must strike to be assured of research funding given the political realities of today's scientific community more than it reflects the sincere assessment of these programs by their authors. If this is the case, I find the behavior of these physicists even more reprehensible than if it were mere willful immodesty. Rather than buying into a system for the allocation of research funding that requires physicists to prevaricate about their own work, and so strengthening the system by lending it the prestige of their cooperation, these scientists should be actively working for its reform, for their own good as well as for the good of physics. 
the best theory of quantum gravity achievable on experimental grounds; until such a moment, there is no scientific warrant for treating any of them as other than they are-extremely tentative hypotheses, in the import of the term as used by Newton when he famously (and I think rightly) proclaimed, Hypotheses non fingo. To treat present day theories of quantum gravity as more would be a signal disservice to the spirit of scientific skepticism and intellectual conscientiousness exemplified by such past revolutionaries as Newton, Maxwell, and Einstein.

A word in parting to those who may think this paper presumptuous and I myself guilty of gross immodesty. Philosophers of science, and of physics in particular, have recently begun discussing issues arising from physicists' work on quantum gravity, but they have tended to accept more or less uncritically the claims of scientific merit for the work made by the physicists themselves. If part of the task of the philosopher of science is to understand what good science is and how it may be practiced, as I do believe - and if one further believes, again as I do, that good science is not merely whatever famous scientists of the day happen to be doing-then the mettle of philosophy of science surely demands that the philosopher speak up against the practice of contemporary physicists when their practice calls for it.

\section{REFERENCES}

Ashtekar, A. (1999), "Quantum mechanics of geometry”, LANL e-archive (xxx.lanl.gov) gr-qc/9901023.

Hawking, Stephen (1975), "Particle creation by black holes", Communications in Mathematical Physics 43: 199-220.

Hooke, Robert ([1672] 1757/1958), "Letter to Henry Oldenburg, Secretary of the Royal Society, containing Hooke's criticism of Newton's doctrine of light", in Newton 1958, 110-115. Originally published in Thomas Birch, The History of the Royal Society of London (London: A. Millar, 1757), Vol. 3, 10-15.

Huygens, Christian ([1673a] 1958), "Letter to Henry Oldenburg, Secretary of the Royal Society, containing Huygen's first criticism of Newton's doctrine of light", in Newton 1958, 136. Originally published in the Philosophical Transactions of the Royal Society 96 (July 21, 1673): 6112.

([1673b] 1958), "Letter to Henry Oldenburg, Secretary of the Royal Society, containing Huygens' second criticism of Newton's doctrine of light", in Newton 1958, 148. Originally published in the Philosophical Transactions of the Royal Society 97 (October 6, 1673): 6112 .

([1690] 1912/1962), Treatise on Light. Translated by S. Thompson. New York: Dover Press. Reprint of the 1912 Macmillan and Co. ed.

Maldacena, Juan (2000), “Gravity, particle physics, and their unification”, LANL e-archive (xxx.lanl.gov) hep-th/0002092.

Maxwell, J.C. ([1864] 1965), "A dynamical theory of the electromagnetic field", in The Scientific Papers of J.C. Maxwell, Vol. 1. New York: Dover, 526-583. Originally read before the Royal Society in December, 1864, and subsequently published in The Royal Society Transactions, Vol. CLV.

Newton, Isaac ([1671/1672] 1958), "Letter of February 6, 1671/1672, to Henry Oldenburg, Secretary of the Royal Society, outlining Newton's researches on light and color", in 
Newton 1958, 116-135. Originally published in the Philosophical Transactions of the Royal Society 80 (February 16, 1671/1672): 3075-3087.

([1672a] 1958), "Letter to Henry Oldenburg, Secretary of the Royal Society, containing Newton's response to Hooke's criticism of Newton's doctrine of light", in Newton 1958, 116-135. Originally published in the Philosophical Transactions of the Royal Society 88 (November 18, 1672): 5084-5103.

([1672b] 1958), "Letter to Henry Oldenburg, Secretary of the Royal Society, containing Newton's response to Pardies' second criticism of Newton's doctrine of light", in Newton 1958, 99-103[106-109]. Originally published in the Philosophical Transactions of the Royal Society 85 (July 15, 1672): 5014-5018. English translation of the original Latin, from which some lines are omitted, originally published in Hutton, Shaw, and Pearson (eds.), Philosophical Transactions of the Royal Society, Abridged, (London, 1809), Vol. 1, 738-743.

([1673a] 1958), "Letter of April 3, 1673, to Henry Oldenburg, Secretary of the Royal Society, containing Newton's response to Huygens' first criticism of Newton's doctrine of light", in Newton 1958, 143-146. Originally published in the Philosophical Transactions of the Royal Society 97 (October 6, 1673): 6108-6111.

- ([1673b] 1958), "Letter to Henry Oldenburg, Secretary of the Royal Society, containing Newton's response to Huygens' second criticism of Newton's doctrine of light", in Newton 1958, 137-142. Originally published in the Philosophical Transactions of the Royal Society 96 (July 21, 1673): 6087-6092.

([1687] 1726/1995), Philosophice Naturalis Principia Mathematica, 3rd ed. (1726). Translated by Andrew Motte, originally published in 1727. Reprint. Amherst, NY: Prometheus Books.

- (1958), Isaac Newton's Papers \& Letters on Natural Philosophy. Edited by I. B. Cohen. Cambridge, MA: Harvard University Press.

Rovelli, Carlo (1998), "Strings, loops and others: A critical survey of the present approaches to quantum gravity", LANL e-archive (xxx.lanl.gov) gr-qc/9803024.

(1999), “Quantum spacetime: What do we know?", LANL e-archive (xxx.lanl.gov) gr-qc/9903045. Reprinted in Craig Callender and Nick Huggett (eds.), Physics Meets Philosophy at the Planck Scale (Cambridge: Cambridge University Press, 2001).

Rovelli, Carlo and L. Smolin (1995), "Discreteness of area and volume in quantum gravity", Nuclear Physics B442: 593-622. Erratum: Nuclear Physics B456 (1995): 734.

Stein, H. (n.d.a), "Further considerations on Newton's method", unpublished manuscript. (n.d.b), "On metaphysics and method in Newton", unpublished manuscript.

Strominger, A. and G. Vafa (1996), "Microscopic origin of the Bekenstein-Hawking entropy", Physical Review Letters B379: 99-104.

Susskind, Leonard (1999), "Strings, black holes and holography", lecture delivered at the Fifth Conference on the History and Foundations of General Relativity [July 8, 1999] at the University of Notre Dame, South Bend, IN.

Wald, R. (1994), Quantum Field Theory in Curved Spacetime and Black Hole Thermodynamics. Chicago Lectures in Physics. Chicago: University of Chicago Press. 